\title{
ON THE REGULARITY OF MINIMIZERS FOR SCALAR INTEGRAL FUNCTIONALS WITH $(p, q)$-GROWTH
}

\author{
PETER BELLA AND MATHIAS SCHÄFFNER
}

\begin{abstract}
We revisit the question of regularity for minimizers of scalar autonomous integral functionals with so-called $(p, q)$-growth. In particular, we establish Lipschitz regularity under the condition $\frac{q}{p}<1+\frac{2}{n-1}$ for $n \geq 3$ which improves a classical result due to Marcellini [JDE'91].
\end{abstract}

\section{Introduction AND MAIN RESUlts}

In this note, we consider the problem of regularity for local minimizers of

$$
\mathcal{F}[u]:=\int_{\Omega} f(\nabla u) d x
$$

where $\Omega \subset \mathbb{R}^{n}, n \geq 2$, is a bounded domain and $f: \mathbb{R}^{n} \rightarrow \mathbb{R}$ is a sufficiently smooth integrand satisfying $(p, q)$-growth of the form

Assumption 1. There exist $0<m \leq M<\infty$ such that

$$
\left\{\begin{array}{l}
z \mapsto f(z) \text { is } C^{2}, \\
m|z|^{p} \leq f(z) \leq M\left(1+|z|^{q}\right), \\
m\left(1+|z|^{2}\right)^{\frac{p-2}{2}}|\lambda|^{2} \leq\left\langle D^{2} f(z) \lambda, \lambda\right\rangle \leq M\left(1+|z|^{2}\right)^{\frac{q-2}{2}}|\lambda|^{2} .
\end{array}\right.
$$

Regularity properties of local minimizer of (1) in the case $p=q$ are classical, see e.g. [14. A systematic regularity theory in the case $p<q$ was initiated by Marcellini in [17, 18. In particular, Marcellini [18, proves

(A) If $2 \leq p<q$ and $\frac{q}{p}<1+\frac{2}{n-2}$ if $n \geq 3$, then every local minimizer $u \in W_{\mathrm{loc}}^{1, q}(\Omega)$ of (1) satisfies $u \in W_{\mathrm{loc}}^{1, \infty}(\Omega)$.

(B) If $2 \leq p<q$ and $\frac{q}{p}<1+\frac{2}{n}$, then every local minimizer $u \in W_{\text {loc }}^{1, p}(\Omega)$ of (1) satisfies $u \in$ $W_{\mathrm{loc}}^{1, \infty}(\Omega)$.

We emphasize that establishing Lipschitz-regularity is the crucial point in the regularity theory for functionals with $(p, q)$-growth in the form (2). Indeed, local boundedness of the gradient implies that the non-standard growth of $f$ and $D^{2} f$ becomes irrelevant and higher regularity (depending on the smoothness of $f$ ) follows by standard arguments, see e.g. [17, Chapter 7] and Corollary 1 below.

By now there is a large and quickly growing literature on regularity results for minimizers of functionals with $(p, q)$-growth, and more general non-standard growth, we refer to 1 , 2, 5, 6, 7, 8, 9, 10, 16, and in particular to 19 for an overview. Under additional structure assumptions on the growth of $f$, for example anisotropic growth of the form

$$
m \sum_{i=1}^{n}\left|z_{i}\right|^{p_{i}} \leq f(z) \leq M \sum_{i=1}^{n}\left(1+\left|z_{i}\right|^{q}\right),
$$

more precise and sharp assumptions on the involved exponents that ensure higher regularity are available in the literature, see e.g. [10, 6]. Moreover, rather sharp conditions are known for certain nonautonomous functionals, see e.g. 1, 5, 9, where also Hölder-continuity of the integrand $f$ in the space variable has to be balanced with $p, q$ and $n$. To the best of our knowledge, there is no improvement of the results (A) and (B) with respect to the relation between the exponents $p, q$ and the dimension 
$n$ available in the literature (without any additional structure assumption). In the present paper, we give such an improvement for $n \geq 3$. Before we state the results, we recall a standard notion of local minimizer in the context of functionals with $(p, q)$-growth

Definition 1. We call $u \in W_{\mathrm{loc}}^{1,1}(\Omega)$ a local minimizer of $\mathcal{F}$ given in (11) iff

$$
f(\nabla u) \in L_{\mathrm{loc}}^{1}(\Omega)
$$

and

$$
\int_{\operatorname{supp} \varphi} f(\nabla u) d x \leq \int_{\operatorname{supp} \varphi} f(\nabla u+\nabla \varphi) d x
$$

for any $\varphi \in W^{1,1}(\Omega)$ satisfying $\operatorname{supp} \varphi \Subset \Omega$.

The main results of the present paper can be summarized as

Theorem 2. Let $\Omega \subset \mathbb{R}^{n}, n \geq 2$ and suppose Assumption 1 is satisfied with $2 \leq p \leq q<\infty$ such that

$$
\frac{q}{p}<1+\frac{2}{n-3} \quad \text { if } n \geq 4
$$

Let $u \in W_{\mathrm{loc}}^{1, q}(\Omega)$ be a local minimizer of the functional $\mathcal{F}$ given in $(\mathbb{1})$. Then, $u \in W_{\mathrm{loc}}^{1, \infty}(\Omega)$.

Theorem 3. Let $\Omega \subset \mathbb{R}^{n}, n \geq 2$ and suppose Assumption 1 is satisfied with $2 \leq p \leq q<\infty$ such that

$$
\frac{q}{p}<1+\min \left\{1, \frac{2}{n-1}\right\} .
$$

Let $u \in W_{\mathrm{loc}}^{1,1}(\Omega)$ be a local minimizer of the functional $\mathcal{F}$ given in (11). Then, $u \in W_{\mathrm{loc}}^{1, \infty}(\Omega)$.

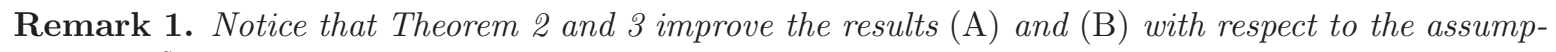
tions on $\frac{q}{p}$ in dimensions $n \geq 3$. The results in [18 apply to more general situations in the sense that: i) (smooth) spatial dependence of $f$ is allowed, ii) a bounded right-hand side is included and iii) nonlinear elliptic equations that not need to be Euler-Lagrange equations of integral functionals of the type (11) are considered. In order to present the new ingredients in the simplest setting we focus the case of autonomous integral functionals with no right-hand side (as in [17]). Very recently [2] sharp criteria for Lipschitz-regularity of minimizers of variational integrals with respect to the right-hand side are obtained under the assumption $\frac{q}{p}<1+\frac{2}{n}$. It is interesting if this can be extended to the case $\frac{q}{p}<1+\frac{2}{n-1}$ if $n \geq 3$.

Remark 2. We do not know if assumptions (3) and (4) are optimal in Theorem 0 and 0 respectively. It is known that Lipschitz-regularity and even boundedness of minimizers fail if $\frac{q}{p}$ is to large depending on the dimension $n$. In particular it is known that in order to ensure boundedness it is necessary that $\frac{q}{p} \rightarrow 1$ if $n \rightarrow \infty$, see [12, 17, 18, 15] for related counterexamples. In particular, it is shown in [15] that the functional

$$
\int_{\Omega}|\nabla u|^{2}+\left|u_{x_{n}}\right|^{4} d x
$$

which satisfies (2) with $p=2$ and $q=4$, admits an unbounded minimizer if $n \geq 6$. Clearly, this does not match condition (4) in Theorem 3 and even not condition (3).

As already mentioned, once boundedness of the gradient is established, higher regularity follows by standard arguments (see e.g. [17, Proof of Theorem D]). Let us state (without proof) a rather direct consequence of Theorem 3

Corollary 1. Let $\Omega \subset \mathbb{R}^{n}, n \geq 2$ and suppose Assumption 1 is satisfied with $2 \leq p \leq q<\infty$ such that (4). Moreover, suppose that $z \mapsto f(z)$ is of class $C_{\text {loc }}^{k, \alpha}$ for some integer $k \geq 2$ and $\alpha \in(0,1)$. Let $u \in W_{\mathrm{loc}}^{1,1}(\Omega)$ be a local minimizer of the functional $\mathcal{F}$ given in $(\mathbb{1})$. Then, $u \in C_{\mathrm{loc}}^{k+2, \alpha}(\Omega)$. 
The proofs of Theorem 2 and 3 are in several aspects quite similar to the approach of Marcellini [17, 18. Following [18, we prove Theorem 2 appealing to the difference quotient method in order to differentiate the Euler-Lagrange equation and use a variant of Moser's iteration argument (see [20]) to prove boundedness of the gradient. The improvement compared to the previous results lies in a recent refinement of Moser's iteration argument in the context of linear non-uniformly elliptic equation obtained by the authors of the present paper in [3] (see 4] for an application to finite difference equations and stochastic analysis). In order to illustrate the relation between Theorem 2 and local boundedness results for non-uniformly elliptic equation, we suppose for the moment that $f$ satisfies (2) with $2=p<q$. A local minimizer $u \in W_{\text {loc }}^{1, q}(\Omega)$ of (1) satisfies the Euler-Lagrange equation

$$
\nabla \cdot D f(\nabla u)=0
$$

and thus by differentiating

$$
\nabla \cdot D^{2} f(\nabla u) \nabla\left(\partial_{j} u\right)=0 \quad \text { for } j=1, \ldots, n .
$$

The coefficient $D^{2} f(\nabla u)$ is non-uniformly elliptic and we have by (2) and the assumption $u \in W_{\text {loc }}^{1, q}(\Omega)$

$$
m|\lambda|^{2} \leq\left\langle D^{2} f(\nabla u) \lambda, \lambda\right\rangle \leq \mu|\lambda|^{2} \quad \text { where } \quad \mu:=M\left(1+|\nabla u|^{2}\right)^{\frac{q-2}{2}} \in L_{\text {loc }}^{\frac{q}{q-2}}(\Omega)
$$

(recall $p=2$ ). Classic regularity results for linear non-uniformly elliptic equations due to Murthy and Stampaccia 21] and Trudinger [22, yield local boundedness of $\partial_{j} u$ if

$$
\frac{q-2}{q}<\frac{2}{n} \Rightarrow \frac{q}{2}<\frac{n}{n-2}=1+\frac{2}{n-2},
$$

which is precisely Marcellini's condition (A) (in the case $p=2$ ). Very recently, the authors of the present paper improved in [3] the assumptions of [21, 22] and established local boundedness and validity of Harnack inequality for linear elliptic equations under essentially optimal assumptions on the integrability of the coefficients, see [11 for related counterexamples. Applied to equation (5), the results of [3] yield local boundedness of $\partial_{j} u$ if

$$
\frac{q-2}{q}<\frac{2}{n-1} \Rightarrow \frac{q}{2}<\frac{n-1}{n-3}=1+\frac{2}{n-3},
$$

which is precisely condition (3). For $p>2$ the results of 3 applied to (5) do not give the claimed condition (31) and thus we need to combine the reasoning of [18] with arguments of [3] and provide an essentially self-contained proof of Theorem 2 Theorem 3 follows from Theorem 2 by a combination of an interpolation argument (similar to [18, Theorem 3.1]) and a suitable approximation procedure (inspired by [8]).

The paper is organized as follows: In Section 2 we recall some results from 18 and present a technical lemma which is used to derive an improved version of Caccioppoli inequality which plays a prominent role in the proof of Theorem 2. In Section 3, we prove Theorem 2 and provide a useful apriori estimate via interpolation, see Corollary 2. Finally, in Section 4, we establish Theorem 2 as a consequence of Corollary 2 and an approximation argument.

\section{Preliminary Lemmas}

For $\alpha \geq 2$ and $k>0$, let $g_{\alpha, k}: \mathbb{R} \rightarrow \mathbb{R}$ be the unique $C^{1}(\mathbb{R})$-function satisfying

$$
g_{\alpha, k}(t)=t\left(1+t^{2}\right)^{\frac{\alpha-2}{2}} \quad \text { for }|t| \leq k,
$$

and which is affine on $\mathbb{R} \backslash\{|t| \leq k\}$. Moreover, we set

$$
G_{\alpha, k}(t):=\frac{g_{\alpha, k}^{2}(t)}{g_{\alpha, k}^{\prime}(t)} .
$$

The following bounds on $G_{\alpha, k}$ are derived in [18] 
Lemma 1 ([18, Lemma 2.6). For every $\alpha \in[2, \infty)$ and $k>0$ there exists $c=c(\alpha, k) \in[1, \infty)$ such that for all $t \in \mathbb{R}$

$$
\begin{aligned}
& G_{\alpha, k}(t) \leq c_{\alpha, k}\left(1+t^{2}\right) \\
& G_{\alpha, k}(t) \leq 2\left(\frac{1+k^{2}}{k^{2}}\right)^{\frac{\alpha-2}{2}}\left(1+t^{2}\right)^{\frac{\alpha}{2}}
\end{aligned}
$$

Appealing to the difference quotient method, it is proven in [18 that local minimizers of (1) satisfying $W_{\text {loc }}^{1, q}(\Omega)$ integrability enjoy higher differentiability.

Lemma 2. Let $\Omega \subset \mathbb{R}^{n}, n \geq 2$ and suppose Assumption 1 is satisfied with $2 \leq p \leq q<\infty$. Let $u \in W_{\mathrm{loc}}^{1, q}(\Omega)$ be a local minimizer of the functional $\mathcal{F}$ given in (11). Then, $u \in W_{\mathrm{loc}}^{2,2}(\Omega)$. Moreover, for every $\eta \in C_{c}^{1}(\Omega)$, any $s \in\{1, \ldots, n\}$ and any $\alpha \geq 2$,

$$
\int_{\Omega} \eta^{2} g_{\alpha, k}^{\prime}\left(u_{x_{s}}\right)\left(1+|\nabla u|^{2}\right)^{\frac{p-2}{2}}\left|\nabla u_{x_{s}}\right|^{2} d x \leq \frac{4 M}{m} \int_{\Omega}|\nabla \eta|^{2} G_{\alpha, k}\left(u_{x_{s}}\right)\left(1+|\nabla u|^{2}\right)^{\frac{q-2}{2}} d x .
$$

Lemma 2 is proven along the lines in [18. However, estimate (10), which is the starting point for our analysis, is not explicitly stated in [18 (as mentioned above, [18, deals with more general equations and additional terms appear on the right-hand side to which our methods do not directly apply) and thus we sketch the proof of Lemma 2 following the reasoning of [18].

Proof of Lemma国. First, we note that since $u \in W_{\mathrm{loc}}^{1, q}(\Omega)$ and $|D f(z)| \leq c(1+|z|)^{q-1}$ for some $c=c(M, n, q) \in[1, \infty)\left(\right.$ by $(2)_{2}$ and $\left.(2)_{3}\right)$, we obtain that $u$ solves the Euler-Lagrange equation

$$
\int_{\Omega}\langle D f(\nabla u), \nabla \varphi\rangle d x=0 \quad \text { for all } \varphi \in W^{1, q}(\Omega) \text { with } \operatorname{supp} \varphi \Subset \Omega .
$$

For $s \in\{1, \ldots, n\}$, we consider the difference quotient operator

$$
\tau_{s, h} v:=\frac{1}{h}\left(v\left(\cdot+h e_{s}\right)-v\right) \quad \text { where } v \in L_{\mathrm{loc}}^{1}\left(\mathbb{R}^{n}\right) .
$$

Fix $\eta \in C_{c}^{1}(\Omega)$. Testing (11) with $\varphi:=\tau_{s,-h}\left(\eta^{2} g_{\alpha, k}\left(\tau_{s, h} u\right)\right)$, we obtain

$$
\begin{aligned}
(I) & :=\int_{\Omega} \eta^{2} g_{\alpha, k}^{\prime}\left(\tau_{s, h} u\right)\left\langle\tau_{s, h} D f(\nabla u), \tau_{s, h} \nabla u\right\rangle d x \\
& =-2 \int_{\Omega} \eta g_{\alpha, k}\left(\tau_{s, h} u\right)\left\langle\tau_{s, h} D f(\nabla u), \nabla \eta\right\rangle d x=:(I I) .
\end{aligned}
$$

Writing $\tau_{s, h} D f(\nabla u)=\left.\frac{1}{h} D f\left(\nabla u+t h \tau_{s, h} \nabla u\right)\right|_{t=0} ^{t=1}$, the fundamental theorem of calculus yields

$$
\begin{aligned}
& \left.\int_{\Omega} \int_{0}^{1} \eta^{2} g_{\alpha, k}^{\prime}\left(\tau_{s, h} u\right)\left\langle D^{2} f\left(\nabla u+t h \tau_{s, h} \nabla u\right)\right) \tau_{s, h} \nabla u, \tau_{s, h} \nabla u\right\rangle d t d x=(I) \\
= & (I I)=-2 \int_{\Omega} \int_{0}^{1} \eta g_{\alpha, k}\left(\tau_{s, h} u\right)\left\langle D^{2} f\left(\nabla u+t h \tau_{s, h} \nabla u\right) \tau_{s, h} \nabla u, \nabla \eta\right\rangle d t d x .
\end{aligned}
$$

Youngs inequality and the definition of $G_{\alpha, k}$, see (7), then yield

$$
|(I I)| \leq \frac{1}{2}(I)+2(I I I),
$$

where

$$
(I I I):=\int_{\Omega} \int_{0}^{1} G_{\alpha, k}\left(\tau_{s, h} u\right)\left\langle D^{2} f\left(\nabla u+t h \tau_{s, h} \nabla u\right) \nabla \eta, \nabla \eta\right\rangle d t d x .
$$

Combining (12), (13) with the assumptions on $D^{2} f$, see (2), we obtain for all $\alpha \geq 2$

$$
\begin{aligned}
& m \int_{\Omega} \int_{0}^{1} \eta^{2} g_{\alpha, k}^{\prime}\left(\tau_{s, h} u\right)\left(1+\left|\nabla u+t h \tau_{s, h} \nabla u\right|^{2}\right)^{\frac{p-2}{2}}\left|\tau_{s, h} \nabla u\right|^{2} d x \leq(I) \\
\leq & 4(I I I) \leq 4 M \int_{\Omega} \int_{0}^{1} G_{\alpha, k}\left(\tau_{s, h} u\right)\left(1+\left|\nabla u+t h \tau_{s, h} \nabla u\right|^{2}\right)^{\frac{q-2}{2}}|\nabla \eta|^{2} d x .
\end{aligned}
$$


Estimate (14) with $\alpha=2$ (and thus $g_{2, k}=t, g_{2, k}^{\prime}=1$ and $G_{2, k}(t)=t^{2}$, see (6), (7)), the assumption $u \in W_{\mathrm{loc}}^{1, q}(\Omega)$ and the arbitrariness of $\eta \in C_{c}^{1}(\Omega)$ and $s \in\{1, \ldots, n\}$ yield $u \in W_{\text {loc }}^{2,2}(\Omega)$. Finally, sending $h$ to zero in (14) we obtain the desired estimate (10) (for this we use that $G_{\alpha, k}$ is quadratic for every $k>0$, see (8), and thus $G_{\alpha, k}\left(\tau_{s, h} u\right) \rightarrow G_{\alpha, k}\left(u_{x_{s}}\right)$ in $L^{\frac{q}{2}}\left(\Omega^{\prime}\right)$ for any $\left.\Omega^{\prime} \Subset \Omega\right)$.

To this point, we essentially recalled notation and statements from [18. Following [18, we will combine (10) with a Moser iteration type argument to establish the desired Lipschitz-estimate. In contrast to 18, we optimize estimate (10) with respect to $\eta$ which will enable us to use Sobolev inequality on spheres instead of balls. The following lemma captures the needed improvement due to a suitable choice of the cut-off function.

Lemma 3. Fix $n \geq 2$. For given $0<\rho<\sigma<\infty$ and $v \in L^{1}\left(B_{\sigma}\right)$ consider

$$
J(\rho, \sigma, v):=\inf \left\{\int_{B_{\sigma}}|v||\nabla \eta|^{2} d x \mid \eta \in C_{0}^{1}\left(B_{\sigma}\right), \eta \geq 0, \eta=1 \text { in } B_{\rho}\right\} .
$$

Then

$$
J(\rho, \sigma, v) \leq(\sigma-\rho)^{-\left(1+\frac{1}{\delta}\right)}\left(\int_{\rho}^{\sigma}\left(\int_{S_{r}}|v|\right)^{\delta} d r\right)^{\frac{1}{\delta}} \quad \text { for every } \delta \in(0,1] .
$$

Proof of Lemma 3 . Estimate (15) follows directly by minimizing among radial symmetric cut-off functions. Indeed, we obviously have for every $\varepsilon \geq 0$

$$
J(\rho, \sigma, v) \leq \inf \left\{\int_{\rho}^{\sigma} \eta^{\prime}(r)^{2}\left(\int_{S_{r}}|v|+\varepsilon\right) d r \mid \eta \in C^{1}(\rho, \sigma), \eta(\rho)=1, \eta(\sigma)=0\right\}=: J_{1 \mathrm{~d}, \varepsilon} .
$$

For $\varepsilon>0$, the one-dimensional minimization problem $J_{1 \mathrm{~d}, \varepsilon}$ can be solved explicitly and we obtain

$$
J_{1 \mathrm{~d}, \varepsilon}=\left(\int_{\rho}^{\sigma}\left(\int_{S_{r}}|v|+\varepsilon\right)^{-1} d r\right)^{-1} .
$$

Let us give an argument for (16). First we observe that using the assumption $v \in L^{1}\left(B_{\sigma}\right)$ and a simple approximation argument we can replace $\eta \in C^{1}(\rho, \sigma)$ with $\eta \in W^{1, \infty}(\rho, \sigma)$ in the definition of $J_{1 \mathrm{~d}, \varepsilon}$. Let $\widetilde{\eta}:[\rho, \sigma] \rightarrow[0, \infty)$ be given by

$$
\widetilde{\eta}(r):=1-\left(\int_{\rho}^{\sigma} b(r)^{-1} d r\right)^{-1} \int_{\rho}^{r} b(r)^{-1} d r, \quad \text { where } b(r):=\int_{S_{r}}|v|+\varepsilon .
$$

Clearly, $\widetilde{\eta} \in W^{1, \infty}(\rho, \sigma)$ (since $b \geq \varepsilon>0$ ), $\widetilde{\eta}(\rho)=1, \widetilde{\eta}(\sigma)=0$, and thus

$$
J_{1 \mathrm{~d}, \varepsilon} \leq \int_{\rho}^{\sigma} \widetilde{\eta}^{\prime}(r)^{2} b(r) d r=\left(\int_{\rho}^{\sigma} b(r)^{-1} d r\right)^{-1} .
$$

The reverse inequality follows by Hölder's inequality: For every $\eta \in W^{1, \infty}(\rho, \sigma)$ satisfying $\eta(\rho)=1$ and $\eta(\sigma)=0$, we have

$$
1=\left(\int_{\rho}^{\sigma} \eta^{\prime}(r) d r\right)^{2} \leq \int_{\rho}^{\sigma} \eta^{\prime}(r)^{2} b(r) d r \int_{\rho}^{\sigma} b(r)^{-1} d r
$$

Clearly, the last two displayed formulas imply (16).

Next, we deduce (15) from (16). For every $s>1$, we obtain by Hölder inequality $\sigma-\rho=\int_{\rho}^{\sigma}\left(\frac{b}{b}\right)^{\frac{s-1}{s}} \leq$ $\left(\int_{\rho}^{\sigma} b^{s-1}\right)^{\frac{1}{s}}\left(\int_{\rho}^{\sigma} \frac{1}{b}\right)^{\frac{s-1}{s}}$ with $b$ as above, and by (16) that

$$
J_{1 \mathrm{~d}, \varepsilon} \leq(\sigma-\rho)^{-\frac{s}{s-1}}\left(\int_{\rho}^{\sigma}\left(\int_{S_{r}}|v|+\varepsilon\right)^{s-1} d r\right)^{\frac{1}{s-1}} .
$$

Sending $\varepsilon$ to zero, we obtain (15) with $\delta=s-1>0$. 


\section{Proof of Theorem 2}

The main result of this section is the following

Theorem 4. Let $\Omega \subset \mathbb{R}^{n}, n \geq 3$, and suppose Assumption 1 is satisfied with $2 \leq p<q<\infty$ such that (3). Fix

$$
\theta=\frac{2 q}{(n-1) p-(n-3) q} \quad \text { if } n \geq 4 \text { and } \quad \theta>\frac{q}{p} \quad \text { if } n=3 .
$$

Let $u \in W_{\mathrm{loc}}^{1, q}(\Omega)$ be a local minimizer of the functional $\mathcal{F}$ given in (1). Then, there exists $c=$ $c(n, m, M, p, q, \theta) \in[1, \infty)$ such that for every $B_{R}\left(x_{0}\right) \Subset \Omega$ and any $\rho \in(0,1)$

$$
\left\|\left(1+|\nabla u|^{2}\right)^{\frac{1}{2}}\right\|_{L^{\infty}\left(B_{\rho R}\left(x_{0}\right)\right)} \leq c((1-\rho) R)^{-n \frac{\theta}{q}}\left\|\left(1+|\nabla u|^{2}\right)^{\frac{1}{2}}\right\|_{L^{q}\left(B_{R}\left(x_{0}\right)\right)}^{\theta} .
$$

Proof of Theorem 2. Theorem 4 contains the claim of Theorem 2 in the case $n \geq 3$ and $2 \leq p<q$. The remaining case $n=2$ is contained in [18, Theorem 2.1] and the statement is classic for $p=q$.

Proof of Theorem 4. Throughout the proof we write $\lesssim$ if $\leq$ holds up to a positive constant which depends only on $n, m, M, p$ and $q$.

Step 1. One step improvement.

Suppose that $B_{2} \Subset \Omega$. We claim that for every

$$
\gamma \in(0,1] \quad \text { satisfying } \frac{n-3}{n-1} \leq \gamma
$$

there exists $c=c(\gamma, n, m, M, p, q) \in[1, \infty)$ such that for every $\frac{1}{2} \leq \rho<\sigma \leq 1$ and any $\alpha \geq 2$

$$
\left\|\phi_{\alpha+p-2}\right\|_{W^{1,2}\left(B_{\rho}\right)}^{2} \leq c \alpha^{2}(\sigma-\rho)^{-\left(1+\frac{1}{\gamma}\right)}\left\|\phi_{(\alpha+q-2) \gamma}\right\|_{W^{1,2}\left(B_{\sigma}\right)}^{\frac{2}{\gamma}}
$$

where we use the shorthand

$$
\phi_{\beta}:=\sum_{j=1}^{n}\left(1+u_{x_{j}}^{2}\right)^{\frac{\beta}{4}} \quad \text { for } \beta>0 .
$$

Moreover, there exists $c=c(n, m, M, p, q) \in[1, \infty)$ such that for every $0<\rho<\sigma \leq 2$ and any $\alpha \geq 2$

$$
\left\|\nabla \phi_{\alpha+p-2}\right\|_{L^{2}\left(B_{\rho}\right)}^{2} \leq c \alpha^{2}(\sigma-\rho)^{-2}\left\|\phi_{\alpha+q-2}\right\|_{L^{2}\left(B_{\sigma}\right)}^{2}
$$

Substep 1.1. We claim that there exists $c=c(\gamma, n, q) \in[1, \infty)$ such that for every $k>0, \alpha \geq 2$, $s \in\{1, \ldots, n\}$, and $\frac{1}{2} \leq \rho<\sigma \leq 1$

$$
\begin{aligned}
I_{\alpha, k, s}(\rho, \sigma): & =\inf \left\{\int_{B_{\sigma}}|\nabla \eta|^{2} G_{\alpha, k}\left(u_{x_{s}}\right)\left(1+|\nabla u|^{2}\right)^{\frac{q-2}{2}} \mid \eta \in C_{0}^{1}\left(B_{\sigma}\right), \eta=1 \text { in } B_{\rho}\right\} \\
& \leq c(\sigma-\rho)^{-\left(1+\frac{1}{\gamma}\right)}\left(\frac{1+k^{2}}{k^{2}}\right)^{\frac{\alpha-2}{2}}\left\|\phi_{(q-2+\alpha) \gamma}\right\|_{W^{1,2}\left(B_{\sigma}\right)}^{\frac{2}{2}} .
\end{aligned}
$$

Assumption $u \in W^{1, q}\left(B_{1}\right)$ and estimate (8) imply that $v:=G_{\alpha, k}\left(u_{x_{s}}\right)\left(1+|\nabla u|^{2}\right)^{\frac{q-2}{2}} \in L^{1}\left(B_{1}\right)$. Hence, Lemma 3 and (9) yield for every $\delta \in(0,1]$

$$
I_{\alpha, k, s}(\rho, \sigma) \leq 2(\sigma-\rho)^{-\left(1+\frac{1}{\delta}\right)}\left(\frac{1+k^{2}}{k^{2}}\right)^{\frac{\alpha-2}{2}}\left(\int_{\rho}^{\sigma}\left(\int_{S_{r}}\left(1+u_{x_{s}}^{2}\right)^{\frac{\alpha}{2}}\left(1+|\nabla u|^{2}\right)^{\frac{q-2}{2}}\right)^{\delta} d r\right)^{\frac{1}{\delta}} .
$$

Appealing to Young's inequality, we find $c=c(n) \in[1, \infty)$ such that

$$
\sum_{j=1}^{n}\left(1+u_{x_{j}}^{2}\right)^{\frac{\alpha}{2}} \sum_{j=1}^{n}\left(1+u_{x_{j}}^{2}\right)^{\frac{q-2}{2}} \leq c \sum_{j=1}^{n}\left(1+u_{x_{j}}^{2}\right)^{\frac{\alpha+q-2}{2}}
$$


(in fact (24) is valid with $c=1+\frac{1}{2} n(n-1)$, see [18, Lemma 2.9]) and thus

$$
\begin{aligned}
\left(1+u_{x_{s}}^{2}\right)^{\frac{\alpha}{2}}\left(1+|\nabla u|^{2}\right)^{\frac{q-2}{2}} & \leq n^{\max \left\{\frac{q-2}{2}-1,0\right\}} \sum_{j=1}^{n}\left(1+u_{x_{j}}^{2}\right)^{\frac{\alpha}{2}} \sum_{j=1}^{n}\left(1+u_{x_{j}}^{2}\right)^{\frac{q-2}{2}} \\
& \stackrel{24}{\leq} c n^{\max \left\{\frac{q-2}{2}-1,0\right\}} \sum_{j=1}^{n}\left(1+u_{x_{j}}^{2}\right)^{\frac{\alpha+q-2}{2}} \\
& \leq c n^{\max \left\{\frac{q-2}{2}-1,0\right\}} \phi_{(\alpha+q-2) \gamma}^{\frac{2}{\gamma}},
\end{aligned}
$$

where in the first inequality we use Jensen's inequality in the case $\frac{q-2}{2} \geq 1$ and the discrete $\ell_{s}-\ell_{1}, s \geq 1$ estimate for $\frac{q-2}{2} \in(0,1)$, and the third inequality is again the discrete $\ell_{s}-\ell_{1}, s \geq 1$ estimate. Hence, we find $c=c(n, q) \in[1, \infty)$ such that

$$
I_{\alpha, k, s}(\rho, \sigma) \leq c(\sigma-\rho)^{-\left(1+\frac{1}{\delta}\right)}\left(\frac{1+k^{2}}{k^{2}}\right)^{\frac{\alpha-2}{2}}\left(\int_{\rho}^{\sigma}\left(\int_{S_{r}} \phi_{(\alpha+q-2) \gamma}^{\frac{2}{\gamma}}\right)^{\delta} d r\right)^{\frac{1}{\delta}} \quad \text { for all } \delta \in(0,1]
$$

To estimate the right-hand side in (25) we use the Sobolev inequality on spheres, i.e for all $\gamma \in(0,1]$ there exists $c=c(n, \gamma) \in[1, \infty)$ such that for every $r>0$

$$
\left(\int_{S_{r}}|\varphi|^{\frac{2}{\gamma}}\right)^{\frac{\gamma}{2}} \leq c\left(\left(\int_{S_{r}}|\nabla \varphi|^{\left(\frac{2}{\gamma}\right)_{*}}\right)^{\frac{1}{\left(\frac{2}{\gamma}\right)_{*}}}+\frac{1}{r}\left(\int_{S_{r}}|\varphi|^{\left(\frac{2}{\gamma}\right)_{*}}\right)^{\frac{1}{\left(\frac{2}{\gamma}\right)_{*}}}\right), \quad \text { where } \frac{1}{\left(\frac{2}{\gamma}\right)_{*}}=\frac{\gamma}{2}+\frac{1}{n-1}
$$

Estimate (26) and assumption (19) in the form $\frac{1}{\left(\frac{2}{\gamma}\right)_{*}}=\frac{\gamma}{2}+\frac{1}{n-1} \frac{n-3}{2(n-1)}+\frac{1}{n-1} \geq \frac{1}{2}$ yield

$$
\begin{aligned}
& \left(\int_{\rho}^{\sigma}\left(\int_{S_{r}} \phi_{(\alpha+q-2) \gamma}^{\frac{2}{\gamma}} d r\right)^{\frac{1}{\delta}}\right. \\
\leq & c\left(\int_{\rho}^{\sigma}\left[\left(\int_{S_{r}}\left|\nabla \phi_{(\alpha+q-2) \gamma}\right|^{\left(\frac{2}{\gamma}\right)_{*}}\right)^{\frac{1}{\left(\frac{2}{\gamma}\right)_{*}}}+\frac{1}{r}\left(\int_{S_{r}} \phi_{(\alpha+q-2) \gamma}^{\left(\frac{2}{\gamma}\right)_{*}}\right)^{\frac{1}{\left(\frac{2}{\gamma}\right) *}}\right]^{\delta \frac{2}{\gamma}} d r\right)^{\frac{1}{\delta}} \\
\leq & \left(\int_{\rho}^{\sigma}\left|S_{r}\right|^{\left(\frac{1}{\left(\frac{2}{\gamma}\right)_{*}}-\frac{1}{2}\right) \frac{2 \delta}{\gamma}}\left[\left(\int_{S_{r}}\left|\nabla \phi_{(\alpha+q-2) \gamma}\right|^{2}\right)^{\frac{1}{2}}+\frac{1}{r}\left(\int_{S_{r}} \phi_{(\alpha+q-2) \gamma}^{2}\right)^{\frac{1}{2}}\right]^{\delta \frac{2}{\gamma}} d r\right)^{\frac{1}{\delta}},
\end{aligned}
$$

where $c=c(\gamma, n) \in[1, \infty)$. Combining (25) and (27) with the choice $\delta=\gamma$, we obtain the claimed estimate (23) (we can ignore the factors $\left|S_{r}\right|$ and $\frac{1}{r}$ in (27) by assumption $\frac{1}{2} \leq \rho<\sigma \leq 1$ ).

Substep 1.2. Proof of (20). Lemma 2 and estimate (23) yield for every $s \in\{1, \ldots, n\}$

$$
\int_{B_{\rho}} g_{\alpha, k}^{\prime}\left(u_{x_{s}}\right)\left(1+|\nabla u|^{2}\right)^{\frac{p-2}{2}}\left|\nabla u_{x_{s}}\right|^{2} d x \leq c(\sigma-\rho)^{-\left(1+\frac{1}{\gamma}\right)}\left(\frac{1+k^{2}}{k^{2}}\right)^{\frac{\alpha-2}{2}}\left\|\phi_{(q-2+\alpha) \gamma}\right\|_{W^{1,2}\left(B_{\sigma}\right)}^{\frac{2}{2}},
$$

where $c=c(\gamma, n, m, M, p, q) \in[1, \infty)$. Sending $k$ to infinity and summing over $s$ from 1 to $n$, we obtain (using $\lim _{k \rightarrow \infty} g_{\alpha, k}^{\prime}(t) \geq\left(1+t^{2}\right)^{\frac{\alpha-2}{2}}$ )

$$
\int_{B_{\rho}} \sum_{j=1}^{n}\left(1+u_{x_{j}}^{2}\right)^{\frac{\alpha+p-4}{2}}\left|\nabla u_{x_{j}}\right|^{2} d x \leq c(\sigma-\rho)^{-\left(1+\frac{1}{\gamma}\right)}\left\|\phi_{(q-2+\alpha) \gamma}\right\|_{W^{1,2}\left(B_{\sigma}\right)}^{\frac{2}{\gamma}} .
$$

Combining the above estimate with the pointwise inequality

$$
\left|\nabla \phi_{\alpha+p-2}\right| \leq \frac{\alpha+p-2}{2} \sum_{j=1}^{n}\left(1+u_{x_{j}}^{2}\right)^{\frac{\alpha+p-4}{4}}\left|\nabla u_{x_{j}}\right|
$$

we obtain that there exists $c=c(\gamma, n, m, M, p, q) \in[1, \infty)$ such that for all $\frac{1}{2} \leq \rho<\sigma \leq 1$ and $\alpha \geq 2$

$$
\left\|\nabla \phi_{\alpha+p-2}\right\|_{L^{2}\left(B_{\rho}\right)}^{2} \leq c \alpha^{2}(\sigma-\rho)^{-\left(1+\frac{1}{\gamma}\right)}\left\|\phi_{(q-2+\alpha) \gamma}\right\|_{W^{1,2}\left(B_{\sigma}\right)}^{\frac{2}{\gamma}} .
$$


It remains to estimate $\left\|\phi_{\alpha+p-2}\right\|_{L^{2}\left(B_{\rho}\right)}$. For this, we use a version of the Poincaré inequality: For every $\varepsilon>0$ there exists $c=c(\varepsilon, n) \in[1, \infty)$ such that for all $r>0$ and $v \in H^{1}\left(B_{r}\right)$

$$
\left(f_{B_{r}}|v|^{2}\right)^{\frac{1}{2}} \leq c\left(r\left(f_{B_{r}}|\nabla v|^{2}\right)^{\frac{1}{2}}+\left(f_{B_{r}}|v|^{\varepsilon}\right)^{\frac{1}{\varepsilon}}\right) .
$$

We recall a proof of (30) at the end of this step. Inequality (30) with $v=\phi_{\alpha+p-2}$ and $\varepsilon=2 \gamma \frac{\alpha+q-2}{\alpha+p-2}$ together with the inequality

$$
1 \leq \phi_{\alpha+p-2} \leq n^{\max \left\{0,1-\frac{\alpha+p-2}{(\alpha+q-2) \gamma}\right\}} \phi_{(\alpha+q-2) \gamma}^{\frac{1}{\gamma} \frac{\alpha+p-2}{\alpha+q-2}}
$$

(the second inequality follows by Jensens inequality if $\frac{(\alpha+q-2) \gamma}{\alpha+p-2} \geq 1$ and the discrete $\ell_{s}-\ell_{1}, s \geq 1$ inequality otherwise) yield

$$
\left\|\phi_{\alpha+p-2}\right\|_{L^{2}\left(B_{\rho}\right)}^{2} \leq c\left(\left\|\nabla \phi_{\alpha+p-2}\right\|_{L^{2}\left(B_{\rho}\right)}^{2}+\left\|\phi_{(\alpha+q-2) \gamma}\right\|_{L^{2}\left(B_{\rho}\right)}^{\frac{2}{\alpha+q-2}}\right),
$$

where $c=c(n, \gamma, p, q) \in[1, \infty)$ (note that $\rho \in\left[\frac{1}{2}, 1\right]$ and $\varepsilon \in\left[2 \gamma, \frac{q}{p} 2 \gamma\right]$ ). The first term on the right-hand side in (31) can be estimated by (29) and the second term (using $p \leq q$ and $\phi_{\beta} \geq 1$ for all $\beta>0$ ) by

$$
\left\|\phi_{(\alpha+q-2) \gamma}\right\|_{L^{2}\left(B_{\rho}\right)}^{\frac{2}{\gamma} \frac{\alpha+p-2}{\alpha+2}} \leq c\left\|\phi_{(\alpha+q-2) \gamma}\right\|_{L^{2}\left(B_{\rho}\right)}^{\frac{2}{\gamma}} .
$$

A combination of (29), (31) and (32) yield (20).

Finally, we recall an argument for (30): Clearly it suffices to proof the statement for $r=1$. Given $\varepsilon>0$, set

$$
U_{\varepsilon}:=\left\{x \in B_{1}:|v(x)| \leq \lambda_{\varepsilon}\right\} \quad \text { where } \quad \lambda_{\varepsilon}:=\left(2 f_{B_{1}}|v|^{\varepsilon}\right)^{\frac{1}{\varepsilon}} .
$$

The choice of $\lambda_{\varepsilon}$ and the Markov inequality yield

$$
\left|B_{1} \backslash U_{\varepsilon}\right| \leq \lambda^{-\varepsilon} \int_{B_{1}}|v|^{\varepsilon} \leq \frac{1}{2}\left|B_{1}\right|
$$

and thus $\left|U_{\varepsilon}\right| \geq \frac{1}{2}\left|B_{1}\right|$. Hence, by a suitable version of the Poincaré inequality, see e.g. [13, eq.(7.45) p. 164], there exists $c=c(n) \in[1, \infty)$ such that

$$
\int_{B_{1}}\left|v-f_{U_{\varepsilon}} v\right|^{2} \leq c \int_{B_{1}}|\nabla v|^{2}
$$

The above inequality, the triangle inequality and

$$
f_{U_{\varepsilon}}|v| \leq 2 \lambda_{\varepsilon}^{1-\varepsilon} f_{B_{1}}|v|^{\varepsilon} \leq 2^{\frac{1}{\varepsilon}}\left(f_{B_{1}}|v|^{\varepsilon}\right)^{\frac{1}{\varepsilon}}
$$

imply (30).

Substep 1.3. Proof of (22). This estimate is an intermediate step in the proof of [18, Lemma 2.10], but for completeness we recall the argument. Lemma 2 with $\eta$ being the affine cutoff function for $B_{\rho}$ in $B_{\sigma}$ yields for every $s \in\{1, \ldots, n\}$

$$
\int_{B_{\rho}} g_{\alpha, k}^{\prime}\left(u_{x_{s}}\right)\left(1+|\nabla u|^{2}\right)^{\frac{p-2}{2}}\left|\nabla u_{x_{s}}\right|^{2} d x \lesssim(\sigma-\rho)^{-2} \int_{B_{\sigma}} G_{\alpha_{k}}\left(u_{x_{s}}\right)\left(1+|\nabla u|^{2}\right)^{\frac{q-2}{2}} d x
$$

and by summing $s$ from 1 to $n$ and sending $k \rightarrow \infty$, we obtain

$$
\int_{B_{\rho}} \sum_{j=1}^{n}\left(1+u_{x_{j}}^{2}\right)^{\frac{\alpha+p-4}{2}}\left|\nabla u_{x_{j}}\right|^{2} d x \lesssim(\sigma-\rho)^{-2} \int_{B_{\sigma}} \phi_{\alpha+q-2}^{2} .
$$

Estimate (22) is a consequence of (28) and (33). 
Step 2. Iteration. Fix $\theta$ as in (17). We claim that there exists $c=c(n, m, M, p, q, \theta) \in[1, \infty)$ such that

$$
\left\|\left(1+|\nabla u|^{2}\right)^{\frac{1}{2}}\right\|_{L^{\infty}\left(B_{\frac{1}{2}}\right)} \leq c\left\|\left(1+|\nabla u|^{2}\right)^{\frac{1}{2}}\right\|_{L^{q}\left(B_{2}\right)}^{\theta} .
$$

Set

$$
\gamma=\frac{n-3}{n-1} \quad \text { if } n \geq 4 \text { and } \quad \gamma=\frac{\frac{p}{q} \theta-1}{\theta-1} \quad \text { if } n=3 .
$$

Note that the assumptions $p<q$ and $\theta>\frac{q}{p}$ yield

$$
0<\gamma<\frac{p}{q} \text { if } n=3
$$

We define a sequence $\left\{a_{k}\right\}_{k \in \mathbb{N}_{0}}$ by

$$
\alpha_{0}:=2, \quad \alpha_{k}:=\frac{1}{\gamma}\left(\alpha_{k-1}+p-2\right)-(q-2) \quad \text { for all } k \in \mathbb{N} .
$$

By induction one sees that

$$
\alpha_{k}=2+\left(\frac{p}{\gamma}-q\right) \sum_{i=0}^{k-1} \gamma^{-i}=2+\left(\frac{p}{\gamma}-q\right) \frac{\gamma^{-k}-1}{\gamma^{-1}-1}=2+p \frac{\gamma^{-k}-1}{1-\gamma}\left(1-\gamma \frac{q}{p}\right) \quad \text { for all } k \in \mathbb{N} .
$$

The choice of $\gamma$ in (35), assumption (3), and (36) together with $p<q$ imply $1-\gamma \frac{q}{p}>0$ and $\gamma^{-1}>1$, hence

$$
\alpha_{k} \rightarrow \infty \quad \text { as } k \rightarrow \infty \text {. }
$$

For $k \in \mathbb{N}$, set $\rho_{k}=\frac{1}{2}+\frac{1}{2^{k+1}}, \sigma_{k}:=\rho_{k}+\frac{1}{2^{k+1}}=\rho_{k-1}\left(\right.$ where $\left.\rho_{0}:=1\right)$, and

$$
A_{k}:=\left\|\phi_{\alpha_{k}+p-2}\right\|_{W^{1,2}\left(B_{\rho_{k}}\right)}^{\frac{2}{\alpha_{+}+p-2}} \text { for all } k \in \mathbb{N}_{0},
$$

where $\phi_{\beta}, \beta \geq 0$ is defined in (21). Since $\alpha_{k-1}+p-2=\left(\alpha_{k}+q-2\right) \gamma$, estimate (20) for $\alpha=\alpha_{k}$ implies

$$
A_{k} \leq\left(c 2^{(k+1)\left(1+\frac{1}{\gamma}\right)} \alpha_{k}^{2}\right)^{\frac{1}{\alpha_{k}+p-2}} A_{k-1}^{\frac{1}{\gamma} \frac{\alpha_{k-1}+p-2}{\alpha_{k}+p-2}} \text { for every } k \in \mathbb{N},
$$

where $c=c(\gamma, n, m, M, p, q) \in[1, \infty)$ as in (20) and thus by iteration

$$
A_{k} \leq A_{0}^{\gamma^{-k}} \prod_{i=1}^{k} \frac{\alpha_{i-1}+p-2}{\alpha_{i}+p-2} \prod_{i=1}^{k}\left(c 2^{(i+1)\left(1+\frac{1}{\gamma}\right)} \alpha_{i}^{2}\right)^{\frac{1}{\alpha_{i}+p-2}} .
$$

Note that for every $k \in \mathbb{N}$

$$
\prod_{i=1}^{k}\left(c 2^{(i+1)\left(1+\frac{1}{\gamma}\right)} \alpha_{i}^{2}\right)^{\frac{1}{\alpha_{i}+p-2}} \leq \exp \left(\sum_{i=1}^{\infty} \frac{\log \left(c 2^{(i+1)\left(1+\frac{1}{\gamma}\right)} \alpha_{i}^{2}\right)}{\alpha_{i}+p-2}\right)=c(\gamma, n, m, M, p, q)<\infty
$$

and

$$
\begin{aligned}
\gamma^{-k} \prod_{i=1}^{k} \frac{\alpha_{i-1}+p-2}{\alpha_{i}+p-2} & =\gamma^{-k} \frac{\alpha_{0}+p-2}{\alpha_{k}+p-2} \\
& =\gamma^{-k} \frac{p}{p \frac{\gamma^{-k}-1}{1-\gamma}\left(1-\gamma \frac{q}{p}\right)+p} \\
& =\left(\frac{\gamma^{-k}}{\gamma^{-k}-1}\right)\left(\frac{1-\gamma}{1-\gamma \frac{q}{p}+\frac{1-\gamma}{\gamma^{-k}-1}}\right) .
\end{aligned}
$$

Hence, sending $k \rightarrow \infty$ in (37), we obtain that there exists $c=c(n, m, M, p, q, \theta) \in[1, \infty)$ (note $\gamma=\gamma(n, p, q, \theta)<1)$ such that

$$
\left\|\left(1+|\nabla u|^{2}\right)^{\frac{1}{2}}\right\|_{L^{\infty}\left(B_{\frac{1}{2}}\right)} \leq c A_{0}^{\frac{1-\gamma}{1-\gamma \frac{g}{p}}}=c\left\|\phi_{p}\right\|_{W^{1,2}\left(B_{1}\right)}^{\frac{2(1-\gamma)}{p-\gamma q}} .
$$


Estimate (22) and $2 \leq p \leq q$ together with $\phi_{\beta} \geq 1$ for all $\beta \geq 0$ yield

$$
\left\|\phi_{p}\right\|_{W^{1,2}\left(B_{1}\right)}^{\frac{2(1-\gamma)}{p-\gamma q}} \lesssim\left\|\phi_{q}\right\|_{L^{2}\left(B_{2}\right)}^{\frac{2(1-\gamma)}{p-\gamma q}} \lesssim\left\|\left(1+|\nabla u|^{2}\right)^{\frac{1}{2}}\right\|_{L^{q}\left(B_{2}\right)}^{\frac{q}{p} \frac{1-\gamma}{1-\gamma}}
$$

Estimates (38), (39) and the choice of $\gamma$ in (35) imply (34).

Step 3. Conclusion. Fix $\rho \in(0,1)$ and $B_{R}\left(x_{0}\right) \Subset \Omega$. By scaling and translation, we deduce from Step 2 that

$$
\left\|\left(1+|\nabla u|^{2}\right)^{\frac{1}{2}}\right\|_{L^{\infty}\left(B_{\frac{R}{4}}\left(x_{0}\right)\right)} \leq c R^{-n \frac{\theta}{q}}\left\|\left(1+|\nabla u|^{2}\right)^{\frac{1}{2}}\right\|_{L^{q}\left(B_{R}\left(x_{0}\right)\right)}^{\theta},
$$

where $c=c(n, m, M, p, q, \theta) \in[1, \infty)$ is the same as in (34). Applying for every $y \in B_{\rho R}\left(x_{0}\right)$ estimate (40) with $B_{R}\left(x_{0}\right)$ replaced by $B_{(1-\rho) R}(y) \subset \Omega$, we obtain

$$
\left\|\left(1+|\nabla u|^{2}\right)^{\frac{1}{2}}\right\|_{L^{\infty}\left(B_{\frac{1-\rho}{4}}(y)\right)} \leq c((1-\rho) R)^{-n \frac{\theta}{q}}\left\|\left(1+|\nabla u|^{2}\right)^{\frac{1}{2}}\right\|_{L^{q}\left(B_{R}\left(x_{0}\right)\right)}^{\theta}
$$

and thus the claimed estimate (18) follows.

By the same interpolation argument as in [18, Theorem 3.1], we deduce from Theorem 4

Corollary 2. Let $\Omega \subset \mathbb{R}^{n}, n \geq 3$ and suppose Assumption 1 is satisfied with $2 \leq p<q<\infty$ such that (44). Let $\theta$ be given as (17) with the additional constrain $\theta<\frac{q}{q-p}$ for $n=3$ and set

$$
\alpha:=\frac{\theta \frac{p}{q}}{1-\theta\left(1-\frac{p}{q}\right)} .
$$

Let $u \in W_{\mathrm{loc}}^{1, q}(\Omega)$ be a local minimizer of the functional $\mathcal{F}$ given in (1). Then, there exists $c=$ $c(n, m, M, p, q, \theta) \in[1, \infty)$ such that for every $B_{2 R}\left(x_{0}\right) \Subset \Omega$

$$
\left\|\left(1+|\nabla u|^{2}\right)^{\frac{1}{2}}\right\|_{L^{\infty}\left(B_{\frac{R}{2}}\left(x_{0}\right)\right)} \leq c R^{-n \frac{\alpha}{p}}\left\|\left(1+|\nabla u|^{2}\right)^{\frac{1}{2}}\right\|_{L^{p}\left(B_{R}\left(x_{0}\right)\right)}^{\alpha} .
$$

Remark 3. A direct calculation yields

$$
\alpha=\frac{2 p}{(n+1) p-(n-1) q} \quad \text { if } n \geq 4 .
$$

For $n=3$, the assumption on $\theta$ in Corollary 0 reads $\theta \in\left(\frac{q}{p}, \frac{q}{q-p}\right)$. Since $2 \leq p<q$, we have

$$
\frac{q}{p}<\frac{q}{q-p} \Leftrightarrow \frac{q}{p}<2,
$$

where the second inequality is ensured by (4) (for $n=3)$.

Proof of Corollary Q We prove the statement for $x_{0}=0$ and $R=1$, the general claim follows by scaling and translation. Throughout the proof we write $\lesssim$ if $\leq$ holds up to a positive constant which depends only on $n, m, M, p, q$ and $\theta$.

For $\nu \in \mathbb{N} \cup\{0\}$, we set $\rho_{\nu}=1-\frac{1}{2^{1+\nu}}$. Combining the elementary interpolation inequality

$$
\left\|\left(1+|\nabla u|^{2}\right)^{\frac{1}{2}}\right\|_{L^{q}\left(B_{\rho_{\nu}}\right)} \leq\left\|\left(1+|\nabla u|^{2}\right)^{\frac{1}{2}}\right\|_{L^{p}\left(B_{\rho_{\nu}}\right)}^{\frac{p}{q}}\left\|\left(1+|\nabla u|^{2}\right)^{\frac{1}{2}}\right\|_{L^{\infty}\left(B_{\rho_{\nu}}\right)}^{1-\frac{p}{q}}
$$

with estimate (18), we obtain for every $\nu \in \mathbb{N}$

$$
\begin{aligned}
\left\|\left(1+|\nabla u|^{2}\right)^{\frac{1}{2}}\right\|_{L^{\infty}\left(B_{\rho_{\nu-1}}\right)} & \stackrel{\sqrt[18]{\lesssim}}{\lesssim}\left(1-\frac{\rho_{\nu-1}}{\rho_{\nu}}\right)^{-n \frac{\theta}{q}}\left\|\left(1+|\nabla u|^{2}\right)^{\frac{1}{2}}\right\|_{L^{q}\left(B\left(\rho_{\nu}\right)\right)}^{\theta} \\
& \stackrel{\sqrt[43]{ }}{\leq}\left(1-\frac{\rho_{\nu-1}}{\rho_{\nu}}\right)^{-n \frac{\theta}{q}}\left\|\left(1+|\nabla u|^{2}\right)^{\frac{1}{2}}\right\|_{L^{p}\left(B\left(\rho_{\nu}\right)\right.}^{\frac{p}{q} \theta}\left\|\left(1+|\nabla u|^{2}\right)^{\frac{1}{2}}\right\|_{L^{\infty}\left(B\left(\rho_{\nu}\right)\right)}^{\left(1-\frac{p}{q}\right) \theta} \\
& \leq c 2^{(1+\nu) n \frac{\theta}{q}}\left\|\left(1+|\nabla u|^{2}\right)^{\frac{1}{2}}\right\|_{L^{p}\left(B_{1}\right)}^{\frac{p}{q} \theta}\left\|\left(1+|\nabla u|^{2}\right)^{\frac{1}{2}}\right\|_{L^{\infty}\left(B_{\rho_{\nu}}\right)}^{\left(1-\frac{p}{q}\right) \theta},
\end{aligned}
$$


where $c=c(n, n, m, M, p, q, \theta) \in[1, \infty)$. Iterating (44) from $\nu=1$ to $\hat{\nu}$, we obtain

$$
\begin{aligned}
& \left\|\left(1+|\nabla u|^{2}\right)^{\frac{1}{2}}\right\|_{L^{\infty}\left(B_{\frac{1}{2}}\right)}=\left\|\left(1+|\nabla u|^{2}\right)^{\frac{1}{2}}\right\|_{L^{\infty}\left(B_{\rho_{0}}\right)} \\
& \stackrel{\text { (44) }}{\leq} 2^{n \frac{\theta}{q} \sum_{\nu=0}^{\hat{\nu}-1}(\nu+1)((1-\gamma) \theta)^{\nu}}\left(c\left\|\left(1+|\nabla u|^{2}\right)^{\frac{1}{2}}\right\|_{L^{p}\left(B_{1}\right)}^{\frac{p}{q} \theta}\right)^{\sum_{\nu=0}^{\hat{\nu}-1}\left(\left(1-\frac{p}{q}\right) \theta\right)^{\nu}}\left\|\left(1+|\nabla u|^{2}\right)^{\frac{1}{2}}\right\|_{L^{\infty}\left(B_{1}\right)}^{\left(\left(1-\frac{p}{q}\right) \theta\right)^{\hat{\nu}}} .
\end{aligned}
$$

The choice of $\theta$ and assumption (4) imply

$$
0<\left(1-\frac{p}{q}\right) \theta<1
$$

Indeed, (46) is ensured for $n=3$ by the assumption $\theta<\frac{q}{q-p}$ and for $n \geq 4$ by

$$
0<\left(1-\frac{p}{q}\right) \theta \stackrel{\sqrt{17}}{=} \frac{2(q-p)}{(n-1) p-(n-3) q}=1-\frac{(n+1) p-(n-1) q}{(n-1) p-(n-3) q} \stackrel{\sqrt[4]{<}}{<} 1 .
$$

Hence, $\sum_{\nu=0}^{\infty}(\nu+1)\left(\left(1-\frac{p}{q}\right) \theta\right)^{\nu} \lesssim 1$ and $\sum_{\nu=0}^{\infty}\left(\left(1-\frac{p}{q}\right) \theta\right)^{\nu}=\frac{1}{1-\theta\left(1-\frac{p}{q}\right)}$. Thus, estimates (18) and (45) yield for every $\hat{\nu} \in \mathbb{N}$

$$
\begin{aligned}
\left\|\left(1+|\nabla u|^{2}\right)^{\frac{1}{2}}\right\|_{L^{\infty}\left(B_{\frac{1}{2}}\right)} & \stackrel{455}{\lesssim}\left\|\left(1+|\nabla u|^{2}\right)^{\frac{1}{2}}\right\|_{L^{p}\left(B_{1}\right)}^{\frac{\theta \frac{p}{q}}{1-\left(1-\frac{p}{q}\right)}}\left\|\left(1+|\nabla u|^{2}\right)^{\frac{1}{2}}\right\|_{L^{\infty}\left(B_{1}\right)}^{\left(\left(1-\frac{p}{q}\right) \theta \hat{\nu}\right.} \\
& \stackrel{(18)}{\lesssim}\left\|\left(1+|\nabla u|^{2}\right)^{\frac{1}{2}}\right\|_{L^{p}\left(B_{1}\right)}^{\alpha}\left\|\left(1+|\nabla u|^{2}\right)^{\frac{1}{2}}\right\|_{L^{q}\left(B_{2}\right)}^{\theta\left(\left(1-\frac{p}{q}\right) \theta\right)^{\hat{\nu}}} .
\end{aligned}
$$

Assumptions $u \in W_{\text {loc }}^{1, q}(\Omega)$ and $B_{2} \Subset \Omega$ imply $\left\|\left(1+|\nabla u|^{2}\right)^{\frac{1}{2}}\right\|_{L^{q}\left(B_{2}\right)}<\infty$ and thus we find $\hat{\nu} \in \mathbb{N}$ such that $\left\|\left(1+|\nabla u|^{2}\right)^{\frac{1}{2}}\right\|_{L^{q}\left(B_{2}\right)}^{\theta\left(\left(1-\frac{p}{q}\right) \theta\right)^{\hat{\nu}}} \leq 2$ which finishes the proof.

\section{Proof of Theorem 3}

The main result of this section is

Theorem 5. Let $\Omega \subset \mathbb{R}^{n}, n \geq 3$ and suppose Assumption 1 is satisfied with $2 \leq p<q<\infty$ such that (41). Let $\theta$ be given as (17) with the additional constrain $\theta<\frac{q}{q-p}$ for $n=3$. Let $u \in W_{\mathrm{loc}}^{1,1}(\Omega)$ be a local minimizer of the functional $\mathcal{F}$ given in (11). Then, there exists $c=c(n, m, M, p, q, \theta) \in[1, \infty)$ such that for every $B_{2 R}\left(x_{0}\right) \Subset \Omega$

$$
\|\nabla u\|_{L^{\infty}\left(B_{\frac{R}{2}}\left(x_{0}\right)\right)} \leq c\left(f_{B_{R}\left(x_{0}\right)} f(\nabla u) d x\right)^{\frac{\alpha}{p}}+1,
$$

where $\alpha$ is given in (41).

Proof of Theorem 3. Theorem [ 5 contains the claim of Theorem 3 in the case $n \geq 3$ and $2 \leq p<q$. The remaining case $n=2$ follows from a combination of [18, Theorem 2.1] with [8, Theorem 2.1], and the result is classic for $p=q$.

Appealing to the a priori estimate of Corollary 2, the statement of Theorem 5 follows with by now well-established approximation arguments. Below, we present a proof of Theorem 5 that closely follows [8, proof of Theorem 2.1, Step 3].

Proof of Theorem 5. Throughout the proof we write $\lesssim$ if $\leq$ holds up to a positive constant which depends only on $n, m, M, p, q$ and $\theta$.

We assume $B_{2} \Subset \Omega$ and show

$$
\|\nabla u\|_{L^{\infty}\left(B_{\frac{1}{8}}\right)} \lesssim\left(f_{B_{1}} f(\nabla u) d x+1\right)^{\frac{\alpha}{p}} .
$$

Clearly the general claim follows by standard scaling, translation and covering arguments. 
Following [8], we introduce two small parameters $\sigma, \varepsilon \in(0,1)$. Parameter $\sigma>0$ is related to a perturbation $f_{\sigma}$ of the integrand $f$

$$
f_{\sigma}(\xi):=f(\xi)+\sigma|\xi|^{q} \quad \text { for every } \xi \in \mathbb{R}^{n} .
$$

Since $f$ satisfies (2) and $\sigma \in(0,1)$, the function $f_{\sigma}$ satisfies (2) with $M$ replaced by $M^{\prime}$ depending on $M$ and $q$. The second parameter $\varepsilon>0$ corresponds to a regularization $u_{\varepsilon}$ of $u$, where $u_{\varepsilon}:=u * \varphi_{\varepsilon}$ with $\varphi_{\varepsilon}:=\varepsilon^{-n} \varphi(\dot{\bar{\varepsilon}})$ and $\varphi$ being a non-negative, radially symmetric mollifier, i.e. it satisfies

$$
\varphi \geq 0, \quad \operatorname{supp} \varphi \subset B_{1}, \quad \int_{\mathbb{R}^{n}} \varphi(x) d x=1, \quad \varphi(\cdot)=\widetilde{\varphi}(|\cdot|) \quad \text { for some } \widetilde{\varphi} \in C^{\infty}(\mathbb{R}) .
$$

Given $\varepsilon, \sigma \in(0,1)$, we denote by $v_{\varepsilon, \sigma} \in u_{\varepsilon}+W_{0}^{1, q}\left(B_{1}\right)$ the unique function satisfying

$$
\int_{B_{1}} f_{\sigma}\left(\nabla v_{\varepsilon, \sigma}\right) d x \leq \int_{B_{1}} f_{\sigma}(\nabla v) d x \quad \text { for all } v \in u_{\varepsilon}+W_{0}^{1, q}\left(B_{1}\right) .
$$

In view of Corollary 2, we have

$$
\begin{aligned}
\left\|\nabla v_{\varepsilon, \sigma}\right\|_{L^{\infty}\left(B_{\frac{1}{8}}\right)} & \stackrel{\sqrt[422]{\lesssim}}{\lesssim}\left(\int_{B_{\frac{1}{4}}}\left|\nabla v_{\varepsilon, \sigma}\right|^{p} d x+1\right)^{\frac{\alpha}{p}} \\
& \stackrel{(2)}{\lesssim}\left(\int_{B_{1}} f_{\sigma}\left(\nabla v_{\varepsilon, \sigma}\right) d x+1\right)^{\frac{\alpha}{p}} \\
\stackrel{(48),(49)}{\leq} & \left(\int_{B_{1}} f\left(\nabla u_{\varepsilon}\right)+\sigma\left|\nabla u_{\varepsilon}\right|^{q} d x+1\right)^{\frac{\alpha}{p}} \\
\leq & \left(\int_{B_{1+\varepsilon}} f(\nabla u) d x+\sigma \int_{B_{1}}\left|\nabla u_{\varepsilon}\right|^{q} d x+1\right)^{\frac{\alpha}{p}},
\end{aligned}
$$

where we used Jensen's inequality and the convexity of $f$ in the last step. Similarly,

$$
\begin{aligned}
m \int_{B_{1}}\left|\nabla v_{\varepsilon, \sigma}\right|^{p} d x & \stackrel{\sqrt[20]{\leq}}{\leq} \int_{B_{1}} f\left(\nabla v_{\varepsilon, \sigma}\right) d x \stackrel{[48]}{\leq} \int_{B_{1}} f\left(\nabla u_{\varepsilon}\right)+\sigma\left|\nabla u_{\varepsilon}\right|^{q} d x \\
& \leq \int_{B_{1+\varepsilon}} f(\nabla u) d x+\sigma \int_{B_{1}}\left|\nabla u_{\varepsilon}\right|^{q} d x .
\end{aligned}
$$

Fix $\varepsilon \in(0,1)$. In view of (50) and (51), we find $w_{\varepsilon} \in u_{\varepsilon}+W_{0}^{1, p}\left(B_{1}\right)$ such that as $\sigma \rightarrow 0$, up to subsequence,

$$
\begin{aligned}
v_{\varepsilon, \sigma} \rightarrow w_{\varepsilon} & \text { weakly in } W^{1, p}\left(B_{1}\right), \\
\nabla v_{\varepsilon, \sigma} \stackrel{*}{\rightarrow} \nabla w_{\varepsilon} & \text { weakly* in } L^{\infty}\left(B_{\frac{1}{8}}\right) .
\end{aligned}
$$

Hence, a combination of (50), (51) with the weak/weak* lower-semicontinuity of convex functionals yield

$$
\begin{aligned}
& \left\|\nabla w_{\varepsilon}\right\|_{L^{\infty}\left(B_{\frac{R}{8}}\right)} \leq \liminf _{\sigma \rightarrow 0}\left\|\nabla v_{\varepsilon, \sigma}\right\|_{L^{\infty}\left(B_{\frac{1}{8}}\right)} \lesssim\left(\int_{B_{1+\varepsilon}} f(\nabla u) d x+1\right)^{\frac{\alpha}{p}} \\
& m \int_{B_{1}}\left|\nabla w_{\varepsilon}\right|^{p} d x \leq \int_{B_{1}} f\left(\nabla w_{\varepsilon}\right) d x \leq \int_{B_{1+\varepsilon}} f(\nabla u) d x .
\end{aligned}
$$

Since $w_{\varepsilon} \in u_{\varepsilon}+W_{0}^{1, q}\left(B_{1}\right)$ and $u_{\varepsilon} \rightarrow u$ in $W^{1, p}\left(B_{1}\right)$, we find by (53) a function $w \in u+W_{0}^{1, p}\left(B_{1}\right)$ such that, up to subsequence,

$$
\nabla w_{\varepsilon} \rightarrow \nabla w \quad \text { weakly in } L^{p}\left(B_{1}\right)
$$


Appealing to the bounds (52), (53) and lower semicontinuity, we obtain

$$
\begin{aligned}
\|\nabla w\|_{L^{\infty}\left(B_{\frac{1}{8}}\right)} & \lesssim\left(\int_{B_{1+\varepsilon}} f(\nabla u) d x+1\right)^{\frac{\alpha}{p}} \\
\int_{B_{1}} f(\nabla w) d x & \leq \int_{B_{1}} f(\nabla u) d x .
\end{aligned}
$$

Inequality (55), the strong convexity of $f$ and the fact $w \in u+W_{0}^{1, p}\left(B_{1}\right)$ imply $w=u$ and thus the claimed estimate (47) is a consequence of (54).

\section{REFERENCES}

[1] P. Baroni, M. Colombo and G. Mingione, Regularity for general functionals with double phase, Calc. Var. Partial Differential Equations 57 (2018), no. 2, Art. 62.

[2] L. Beck and G. Mingione, Optimal Lipschitz criteria and local estimates for non-uniformly elliptic problems, Preprint (2018), available at https://arxiv.org/abs/1806.03326.

[3] P. Bella and M. Schäffner, Local boundedness and Harnack inequality for solutions of linear non-uniformly elliptic equations. Preprint (2019). Available at https://arxiv.org/abs/1901.07958.

[4] P. Bella and M. Schäffner, Quenched invariance principle for random walks among random degenerate conductances, To appear in Ann. Probab. Available at https://arxiv.org/abs/1902.05793

[5] M. Colombo and G. Mingione, Regularity for double phase variational problems, Arch. Ration. Mech. Anal. 215 (2015), no. 2, 443-496.

[6] G. Cupini, P. Marcellini and E. Mascolo, Local boundedness of minimizers with limit growth conditions, J. Optimization Th. Appl. 166 (2015), 1-22.

[7] M. Eleuteri, P. Marcellini and E. Mascolo, Regularity for scalar integrals without structure conditions, To appear Adv. Calc. Car. Available at https://doi.org/10.1515/acv-2017-0037.

[8] L. Esposito, F. Leonetti and G. Mingione, Higher integrability for minimizers of integral functionals with $(p, q)$ growth, J. Differential Equations 157 (1999), no. 2, 414-438.

[9] L. Esposito, F. Leonetti and G. Mingione, Sharp regularity for functionals with $(p, q)$ growth, J. Differential Equations 204 (2004), no. 1, 5-55.

[10] N. Fusco and C. Sbordone, Some remarks on the regularity of minima of anisotropic integrals, Commun. Partial Diff. Equ. 18 (1993), 153-167.

[11] B. Franchi, R. Serapioni and F. Serra Cassano, Irregular solutions of linear degenerate elliptic equations, Potential Anal. 9 (1998), no. 3, 201-216.

[12] M. Giaquinta, Growth conditions and regularity, a counterexample, Manuscripta Math. 59 (1987), no. 2, $245-248$.

[13] D. Gilbarg and N. Trudinger, Elliptic partial differential equations of second order, Springer, 1998.

[14] E. Giusti, Direct methods in the calculus of variations, World Scientific Publishing Co., Inc., River Edge, NJ, 2003. viii $+403 \mathrm{pp}$

[15] M. C. Hong, Some remarks on the minimizers of variational integrals with nonstandard growth conditions, Boll. Un. Mat. Ital. A (7) 6 (1992), no. 1, 91-101.

[16] G. M. Lieberman, The natural generalization of the natural conditions of Ladyzhenskaya and Uraltseva for elliptic equations, Commun. Partial Differ. Equations 16 (1991), 311-361.

[17] P. Marcellini, Regularity of minimizers of integrals of the calculus of variations with nonstandard growth conditions, Arch. Rational Mech. Anal. 105 (1989), no. 3, 267-284.

[18] P. Marcellini, Regularity and existence of solutions of elliptic equations with $p, q$-growth conditions, J. Differential Equations 90 (1991), no. 1, 1-30.

[19] G. Mingione, Regularity of minima: an invitation to the dark side of the calculus of variations, Appl. Math. 51 (2006), no. 4, 355-426.

[20] J. Moser, A new proof of De Giorgi's theorem concerning the regularity problem for elliptic differential equations, Comm. Pure Appl. Math. 13 (1960), 457-468.

[21] M. K. V. Murthy and G. Stampacchia, Boundary value problems for some degenerate-elliptic operators, Ann. Mat. Pura Appl. (4) 80 (1968), 1-122.

[22] N. S. Trudinger, On the regularity of generalized solutions of linear, non-uniformly elliptic equations, Arch. Rational Mech. Anal. 42 (1971), 50-62.

TU Dortmund, Fakultät für Mathematik, Lehrstuhl I, Vogelpothsweg 87, 44227 Dortmund, Germany.

E-mail address: peter.bella@math.tu-dortmund.de

Mathematisches Institut Universität Leipzig, Augustusplatz 10, 04103 Leipzig, Germany.

E-mail address: schaeffner@math.uni-leipzig.de 